\title{
Effect of Partial Shrouds on the Performance and Flow Field of a Low-Aspect-Ratio Axial-Flow Fan Rotor
}

\author{
N. Sitaram and G. Ch. V. Sivakumar \\ Thermal Turbomachines Laboratory, Department of Mechanical Engineering, Indian Institute of Technology Madras, \\ Chennai 600 036, India \\ Correspondence should be addressed to N. Sitaram, nsitaram@iitm.ac.in
}

Received 2 April 2011; Accepted 16 August 2011

Academic Editor: David Japikse

Copyright ( $\odot 2011$ N. Sitaram and G. Ch. V. Sivakumar. This is an open access article distributed under the Creative Commons Attribution License, which permits unrestricted use, distribution, and reproduction in any medium, provided the original work is properly cited.

\begin{abstract}
The flow field at the rotor exit of a low aspect ratio axial flow fan for different tip geometries and for different flow coefficients is measured in the present study. The following configurations are tested: (1) rotor without partial shroud, designated as rotor (wos), (2) rotor with partial shroud, designated as rotor (ws), and (3) rotor with perforated (perforations in the shape of discrete circular holes) partial shroud, designated as rotor (wps). From steady state measurements, the performance of rotor (wps) is found to be the best. Both the rotors with partial shrouds have stalled at a higher flow coefficient compared to that of rotor (wos). From periodic flow measurements, it is concluded that the low velocity region near the tip section is considerably reduced with the use of partial shrouds with perforations. The extent of this low velocity region for both rotor (wos) and rotor (wps) increases with decreasing flow coefficient due to increased stage loading. This core of low momentum fluid has moved inwards of the annulus and towards the pressure side as the flow coefficient decreases. The extent of the low momentum fluid is smaller for rotor (wps) than that of rotor (wos) at all flow coefficients.
\end{abstract}

\section{Introduction}

Leakage of flow around the tip of unshrouded rotor blades is inevitable in turbomachines. The performance of a fan or compressor is sensitive to the tip region geometry and flow in this region, as it affects the flow over much of the blade span. It is well known fact that the end wall losses comprise a substantial proportion of the total losses, particularly in low-aspect-ratio turbomachines. A significant fraction of the end wall losses is attributed to the tip leakage flow and its interaction with the secondary flow, blade boundary layer, annulus wall boundary layer, and the scraping vortex. The dominant source of leakage loss is due to the mixing of the leakage vortex with the primary flow, which has passed through the blades. As the two flows have different velocities and temperatures, the mixing process generates entropy. Most of the earlier research was confined to understand this flow field in large-aspect-ratio rotors (Hunter and Cumpsty [1], Lakshminarayana et al. [2], Inoue and Kuroumaru [3], and Lakshminarayana et al. [4]). A comprehensive review of the investigations on the turbomachinery tip clearance effects is given by Peacock [5].

Several techniques are tried to improve the performance of axial flow fans and compressors with respect to increasing stall margin and relaxing tip clearance. The manufacture of rotors with small clearances is expensive, and there is also a possibility of blade damage at small clearance. Hence it looks advantageous to manufacture rotors with larger clearances but with performance and operating range equivalent to that at smaller clearances. This can be achieved by active and passive techniques. These techniques generally involve some form of casing treatment near the rotor tip for the purpose of delaying rotor tip stall or reducing the detrimental effects resulting from excessive tip clearance. These techniques are casing treatment [6], vortex generators on the casing wall and rotor blades [7], recessing the casing over the rotor tips [8], recessed vane casing [9], and shrouding of the blades. In addition many active techniques are used to control tip clearance flows. Some of these are air injection [10] and plasma actuation [11]. 


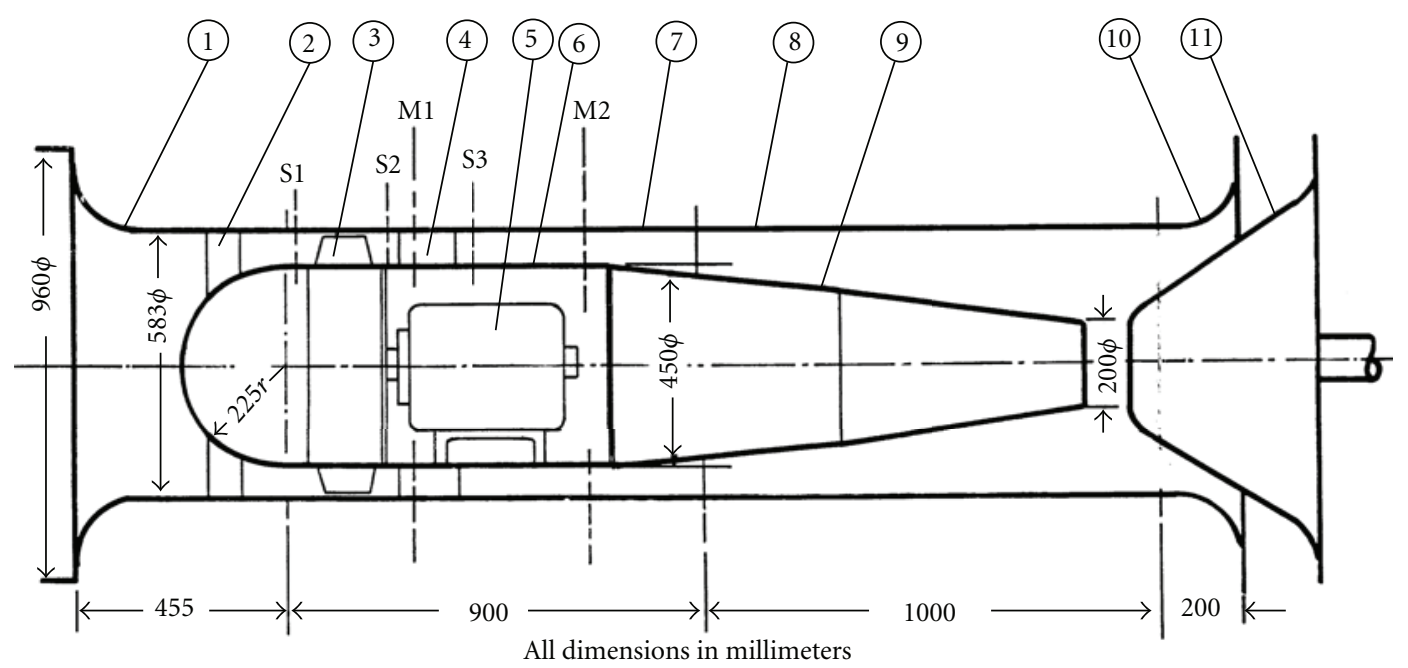
(1) Inlet nozzle
(7) Casing
(2) Radial supports for hub nose cone
(8) Exit duct
(3) Rotor
(9) Conical hub
(4) Stator
(10) Exit nozzle
(5) A.C. motor
(11) Throttle cone
(6) Motor housing

M1 and M2: location of stay rods for motor housing

S1, S2, and S3: traverse stations for probes

FIGURE 1: Schematic of the low-aspect-ratio axial-flow fan facility.

Partial shrouds may provide a significant aerodynamic performance gain. This gain is expected because the leakage flow, initiated near the leading edge on the blade pressure surface, would travel a longer distance over the width of the shroud during which it is forced further downstream. On emerging from the tip clearance, leakage flow interaction with the passage flow near the tip suction surface takes place further downstream where its harmful effects are reduced.

Patel [12] conducted tests with and without partial shrouds on the rotor blades of a single stage axial gas generator turbine at different values of tip clearance. He observed 1.2-point improvement in total efficiency with partial shrouds. But he found that the sensitivity of performance to the tip clearance remains unaltered by partial shrouds. Ishida et al. [13] found that a partial shroud attached to the tip of a rotor blade of a centrifugal blower improved its performance. Corsini et al. [14] had carried out extensive investigations on axial flow fans with partial shrouds with the objective of reducing noise. Akturk and Camci [15] measured three-dimensional mean flow near the tip of a ducted axial fan rotor using a stereoscopic particle image velocimeter. A number of novel tip treatments based on custom-designed pressure side extensions were tested to mitigate tip leakage flow. The chordwise location and the width of the extension in the circumferential direction are systematically varied. Tip platform extensions near the rotor blade trailing edge were found to show the best tip treatment performance. They $[16,17]$ further carried out computational and experimental investigations on additional tip treatments. The treatments included tip platform extensions on the pressure side of the rotor blade at its tip and squealers on the rotor blade tip. These treatments were tested individually and in combination. The squealers are extended in full from the rotor blade leading edge to the trailing edge on the tip and partially from the middle of the rotor blade leading edge to the trailing edge on the tip. Both straight and inclined full squealer treatments provided the best performance. By using these treatments, rotor blades at larger clearance can be used with performance and operating range equivalent to that at smaller clearances and without risk of damage to the rotor blades. Hence the tip clearance can be relaxed reducing manufacturing costs.

The present investigation has similar objective but to improve the aerodynamic performance of an axial flow fan. The present investigation explores an alternative method to improve the performance of an axial flow rotor. The alternative method consists of providing perforations in the shape of discrete circular holes on the partial shroud.

\section{Motivation and Objective}

The flow in the low-aspect-ratio axial flow compressors is very complex and highly three-dimensional. The flow at the exit of the rotor is highly nonuniform in both axial and 
TABLE 1: Details of the low-aspect-ratio axial-flow fan.

\begin{tabular}{|c|c|c|c|}
\hline Design volume flow, $V$ & $2.5 \mathrm{~m}^{3} / \mathrm{s}$ & Specific work, $W$ & $650 \mathrm{~m}^{2} / \mathrm{s}^{2}$ \\
\hline Speed, $n$ & $1450 \mathrm{rpm}$ & Shape number, $N_{s h}=n \sqrt{ } V /\left(60 W^{0.75}\right)$ & 0.30 \\
\hline Design flow coefficient, $\phi_{d}$ & 0.52 & Design pressure rise coefficient, $\psi_{d}$ & 0.58 \\
\hline Rotor hub diameter, $d_{h}$ & $450 \mathrm{~mm}$ & Rotor tip diameter, $d_{t}$ & $580 \mathrm{~mm}$ \\
\hline Casing inner diameter, $d_{c}$ & $583 \mathrm{~mm}$ & Rotor tip clearance, $t(t / C h * 100 \%)$ & $1.5 \mathrm{~mm}(1.1 \%)$ \\
\hline Number of rotor blades, $Z_{R}$ & 14 & Rotor aspect ratio, $\mathrm{AR}_{R}$ & 0.48 \\
\hline NACA 0008 type FRP rotor blades & - & Free vortex design & - \\
\hline Number of stator blades, $Z_{S}$ & 13 & Stator aspect ratio, $\mathrm{AR}_{S}$ & 0.46 \\
\hline Circular arc sheet metal stator blades & - & Stator chord, $\mathrm{Ch}_{S}$ & $145 \mathrm{~mm}$ \\
\hline Stator inlet angle, $\alpha_{2 b}$ & $54^{\circ}$ & Stator exit angle, $\alpha_{3 b}$ & $100^{\circ}$ \\
\hline
\end{tabular}

(All angles are w.r.t. tangential direction).

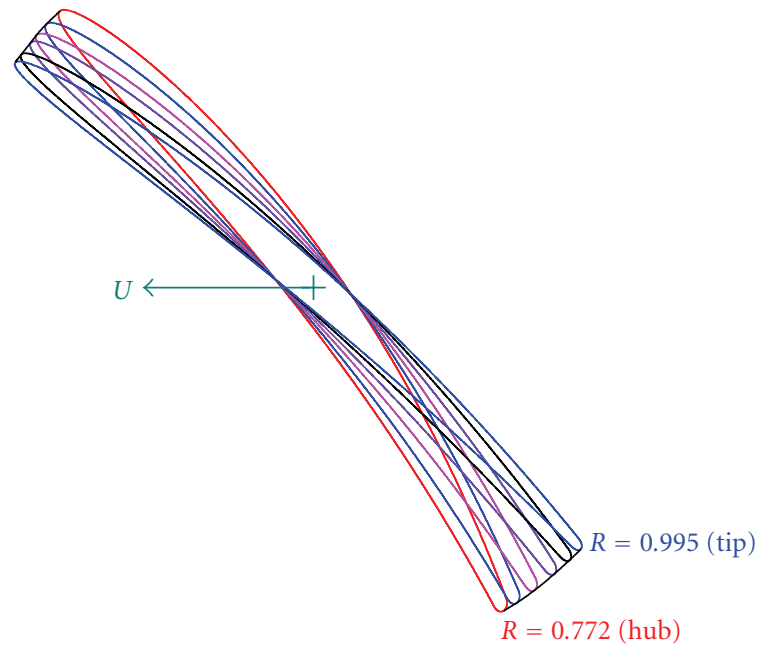

FIGURE 2: Rotor blade profiles at various radial stations.

circumferential directions and highly turbulent. Low-aspectratio axial flow compressors have higher loading levels, higher efficiency, better stability, and vibrational characteristics (Wennerstrom [18]). They are more distortion tolerant due to larger chords of the rotor blades. Such compressors find applications in the rear stages of multistage axial compressors in aircraft gas turbine engines and for ventilation purposes. There are relatively few investigations on low-aspect-ratio compressor aerodynamics in spite of their importance. Because of large values of relative tip clearance (tip clearance/ blade span), the clearance losses in low-aspect-ratio axialflow compressors are expected to be large. The objective of the present research is to investigate the effectiveness of the partial shrouds attached to the tip of the rotor blades of lowaspect-ratio axial-flow fan to reduce the tip clearance losses. In addition, an alternative method of providing perforations in the shape of discrete circular holes on the partial shroud is explored to further improve the performance of the rotor.

\section{Experimental Facility and Instrumentation}

3.1. Experimental Facility. The schematic layout of the test facility is shown in Figure 1. The design details of the fan are given in Table 1. The details of the rotor blade geometry are
TABLE 2: Details of rotor blades.

\begin{tabular}{lccclc}
\hline $\begin{array}{l}\text { Radius } \\
\text { ratio, } R\end{array}$ & $\beta_{1 b}(\mathrm{Deg})$ & $\beta_{2 b}(\mathrm{Deg})$ & $t_{\max } / \mathrm{Ch}(\%)$ & $\begin{array}{l}\mathrm{Ch} \\
(\mathrm{mm})\end{array}$ & $S(\mathrm{~mm})$ \\
\hline 0.772 & 35.2 & 59.5 & 7.500 & 136 & 101.0 \\
0.796 & 34.4 & 57.6 & 7.285 & 136 & 104.1 \\
0.818 & 33.6 & 55.7 & 7.087 & 136 & 107.0 \\
0.842 & 32.9 & 54.0 & 6.872 & 136 & 110.2 \\
0.866 & 32.2 & 52.3 & 6.657 & 136 & 113.3 \\
0.890 & 31.5 & 50.7 & 6.442 & 136 & 116.5 \\
0.913 & 30.8 & 49.1 & 6.235 & 136 & 119.4 \\
0.937 & 30.2 & 47.6 & 6.020 & 136 & 122.5 \\
0.961 & 29.5 & 46.2 & 5.805 & 136 & 125.7 \\
0.985 & 29.0 & 44.8 & 5.590 & 136 & 128.8 \\
0.995 & 28.4 & 43.5 & 5.500 & 136 & 130.8 \\
\hline
\end{tabular}

(All angles are w.r.t. tangential direction).

given in Table 2. Rotor blade profiles at alternate radial stations from rotor hub to tip are shown in Figure 2.

3.2. Partial Shrouds. The configurations studied are rotor without partial shroud, designated as rotor (wos), rotor with partial shroud, designated as rotor (ws), and rotor with perforated partial shroud, designated as rotor (wps) to permit small amount of leakage flow to leak over the tip. The partial shroud attached to the rotor tip is shown in Figure 3. Inlet and exit blade angles with respect to the tangential direction are shown in Figure 3. The details of the partial shroud are given below.

The partial shroud is made from a $0.2 \mathrm{~mm}$ thick stainless steel sheet. The sheet is rolled to the rotor tip diameter, and then the profiles matching the required partial shroud are cut. These profiled shrouds are pasted onto the tip of the blade using a very thin layer of Araldite. This method seemed to be satisfactory as no partial shroud came off with the rotor operating at the design speed of $1450 \mathrm{rpm}$. Also, no vibrations of the partial shrouds were observed from the stroboscope visualizations. Care is taken so that there are no burrs at the edges of the shroud, which otherwise would cause frictional losses. The shape of the shroud is chosen as aerofoil corresponding to the tip section, but with $11 \%$ maximum thickness, which is equal to twice the maximum thickness of 


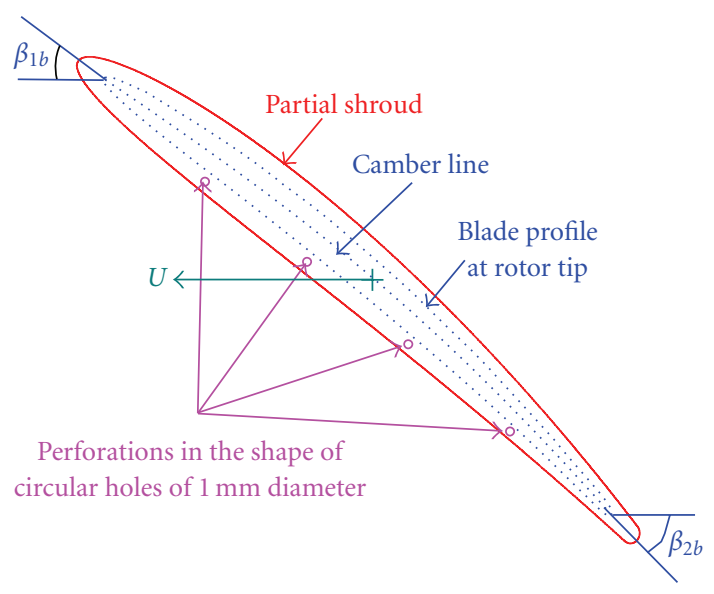

Figure 3: Rotor Blade Tip Profile with Partial Shroud.

the rotor blade tip section. The partial shroud is extended about $5 \mathrm{~mm}$ downstream of the rotor blade leading edge and about $5 \mathrm{~mm}$ upstream of the rotor blade trailing edge. As the tip leakage flow has its maximum velocity occurring at midchord, maximum extension from the tip profile is given at this position. This shroud is slightly overcurved than the meridional curvature of the blade tip. Care is taken so that the tip clearance remains the same at $\tau=1.1 \%$ with and without partial shroud. This is achieved by reducing the radius of the rotor tip by $0.2 \mathrm{~mm}$ to account for the thickness of the partial shroud. Perforations in the shape of discrete circular holes of $1 \mathrm{~mm}$ diameter are made on the pressure side of the partial shroud, to explore the possibility of improving the rotor performance. Four holes are provided at $20 \%, 40 \%, 60 \%$, and $80 \%$ of the rotor blade chord at the tip. Although tip leakage vortex starts near the rotor blade tip leading edge, its strength is visible at around 20\% chord and its strength is maximum at about midchord. The holes are located in between the rotor blade pressure surface edge and the edge of the partial shroud. There is a lot of scope to experiment with the size, number, and location of the holes.

3.3. Steady State Measurements. The inlet flow is measured with a cobra probe. A precalibrated miniature five hole probe (head diameter: $2.5 \mathrm{~mm}$ ) is used to measure flow at the exit of the rotor. The probe is traversed in the radial direction, and measurements are taken at twenty radial locations for four flow coefficients, namely, $\phi=0.42$, near stall flow coefficient, $\phi=0.48$, below design flow coefficient, $\phi=0.55$, near design flow coefficient, and $\phi=0.64$, near maximum flow coefficient. A scanning box (Model FCO 91-3) and a micromanom-e-ter (Model FCO012, range: $\pm 199 \mathrm{~mm}$ of water gage and accuracy of $0.1 \mathrm{~mm}$ water gage) manufactured by Furness Controls, Bexhill, UK are used for the pressure probe measurements.

3.4. Periodic Flow Measurements. A slanted single sensor hot wire probe (DANTEC miniature hot wire probe 55P12) is used to measure the periodic and highly three dimensional flow at the rotor exit. The method of Brunn et al. [19] is used
TABLE 3: Uncertainty in five hole probe measurements.

\begin{tabular}{lccc}
\hline Parameter & Bias error & Precision error & Total error \\
\hline Pressure & $\pm 0.45 \%$ & $\pm 2.00 \%$ & $\pm 2.05 \%$ \\
Velocity & $\pm 1.11 \%$ & $\pm 2.00 \%$ & $\pm 2.29 \%$ \\
Axial velocity & $\pm 1.41 \%$ & $\pm 4.70 \%$ & $\pm 4.90 \%$ \\
Tangential velocity & $\pm 1.41 \%$ & $\pm 4.70 \%$ & $\pm 4.90 \%$ \\
Radial velocity & $\pm 1.41 \%$ & $\pm 4.70 \%$ & $\pm 4.90 \%$ \\
Flow angle & $\pm 0.61 \%$ & $\pm 3.00 \%$ & $\pm 3.07 \%$ \\
Radial angle & $\pm 0.61 \%$ & $\pm 3.00 \%$ & $\pm 3.07 \%$ \\
\hline
\end{tabular}

to derive velocity components from the voltages measured at eight positions.

The probe is rotated in eight positions about its axis and from the measured mean voltages of the probe in these positions; the three mean velocity components are derived. There are several ways of extracting the information on the periodic (or pitchwise) distribution of the velocity components. The technique termed as phase locked ensemble averaging technique (PLEAT) [20] is used to acquire the pitchwise distribution of velocities. In this technique, the hot-wire signal is triggered by a magnetic pickup at a fixed point during each revolution. Corresponding points in successive wave forms are then averaged with one another so that only components repeating every rotor revolution are retained. The hot wire probe signal is recorded by a 16-bit ADVANTEST R-9211A signal analyzer and stored for further processing. A magnetic pick up on the fan shaft producing 1 pulse/revolution is used to trigger the start of data acquisition. The instrumentation for the periodic flow measurements is schematically shown in Figure 4.

Measurements are carried out at three flow coefficients: namely, at $\phi=0.48,0.55$, and 0.64 at the rotor exit at a nondimensional axial distance of 1.50 times the axial chord at the rotor tip and at 14 radial locations. The radial distance is nondimensionalised by $r_{c}$ and pitchwise distance, by blade spacing. The velocities are nondimensionalised with respect to the imaginary blade speed based on the casing radius, that is, $U_{c}$.

3.5. Error Estimates. Measurements by both five hole probes and hot wire probes suffers from many sources of errors. The uncertainty in these measurements is estimated using the analysis proposed by Moffat [21]. The total error of each instrument used for a particular measurement is split into fixed or bias error and random or precision error. The bias error is given by the manufacturer of the instrument and the precision error can be reduced by increasing the number of samples taken. The total error in the measurement of any flow parameter is given by the sum of the errors of all the instruments used. Based on this procedure, the uncertainty in the measurements of five hole and hot wire probes are given in Tables 3 and 4, respectively.

In addition, five hole probes suffer from large errors near solid surfaces and in case of flows with large pressure or velocity gradients. Hence the uncertainty in the measurements with five hole probes near the hub and casing is 


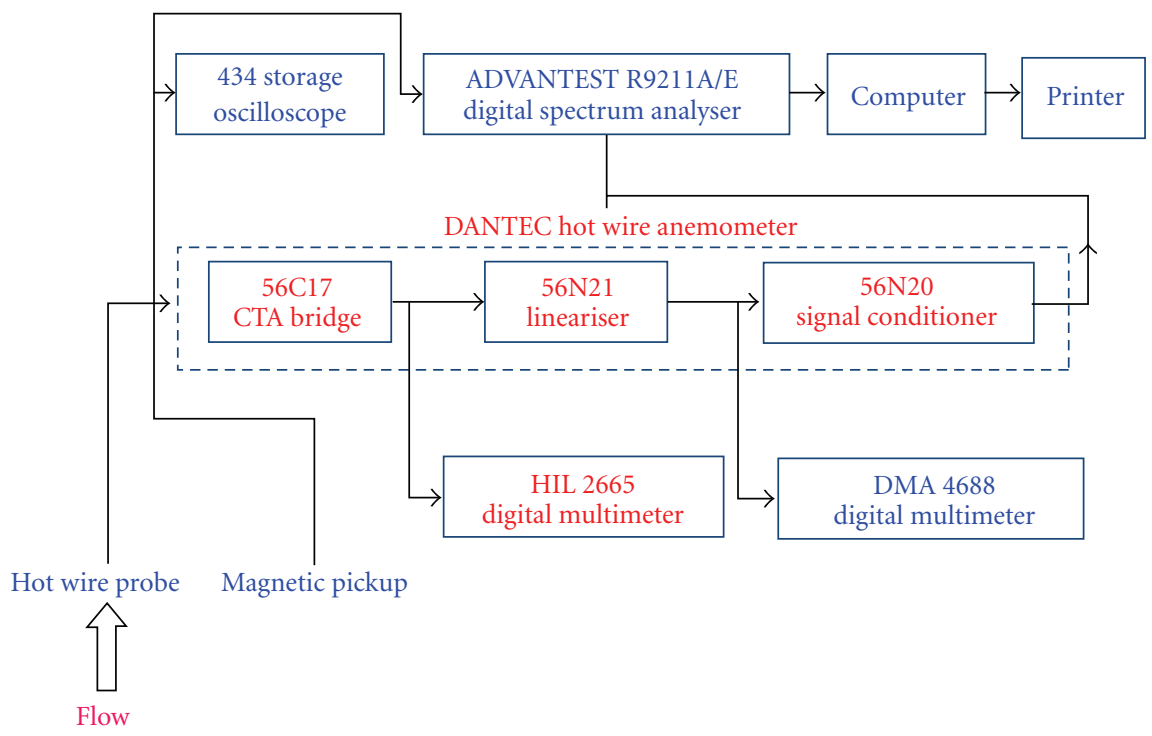

FIGURE 4: Schematic of instrumentation for hot wire probe measurements.

TABLE 4: Uncertainty in hot wire probe measurements.

\begin{tabular}{lccc}
\hline Parameter & Bias error & Precision error & Total error \\
\hline Velocity & $\pm 2.99 \%$ & $\pm 1.23 \%$ & $\pm 3.23 \%$ \\
Axial velocity & $\pm 1.43 \%$ & $\pm 0.58 \%$ & $\pm 1.54 \%$ \\
Tangential velocity & $\pm 2.21 \%$ & $\pm 0.92 \%$ & $\pm 2.39 \%$ \\
Radial velocity & $\pm 1.43 \%$ & $\pm 0.58 \%$ & $\pm 1.54 \%$ \\
\hline
\end{tabular}

higher than the quoted values in Table 3. Similar to five hole probes, hot wire probes also suffer from large errors near solid surfaces and in flows with large pressure or velocity gradients. Hence the uncertainty in the measurements with hot wire probes near the hub and casing and in the wakes is higher than the quoted values in Table 4.

Detailed uncertainty in the measurements with five hole and hot wire probes is presented in Siva Kumar [22].

\section{Results and Discussion}

Results from the present investigation are presented and interpreted in this section. Results obtained from the pressure probe measurements are presented first followed by the results obtained from hot wire probe measurements. Mass-averaged values obtained from these measurements are also presented in this section.

\subsection{Pressure Probe Measurements}

\subsubsection{Radial Distribution of Flow Parameters at the Rotor Exit}

Total Pressure Coefficient, $\psi_{02}$. The distribution of total pressure coefficient for the four flow coefficients and for the three configurations at the rotor exit is presented in Figure 5.

There is substantial increase in total pressure for the rotor (ws) compared to that of rotor (wos) at $\phi=0.64$, but very

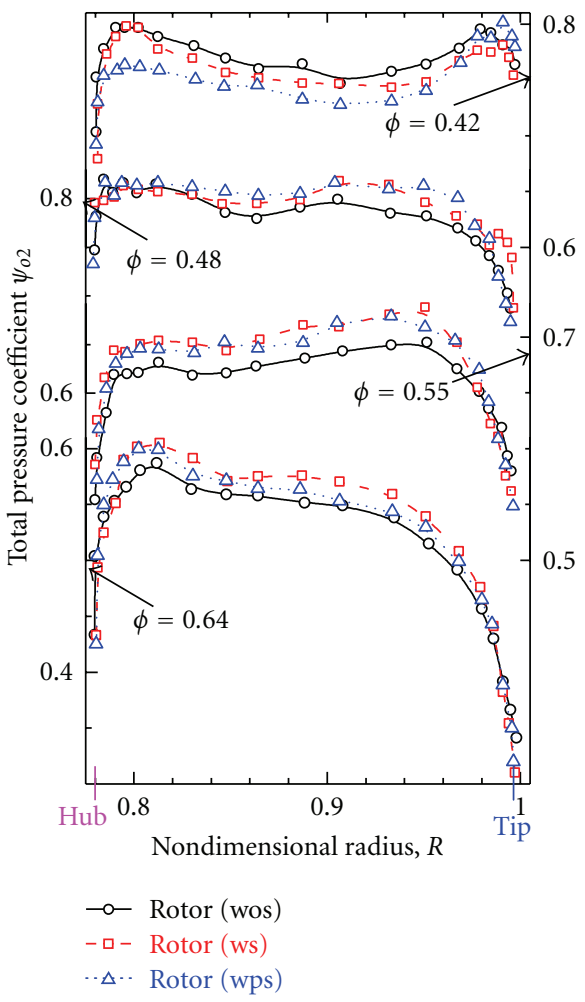

FIGURE 5: Radial distribution of total pressure coefficient at the rotor exit.

nominal increase is observed for rotor (wps). But for $\phi=$ 0.55 and 0.48 there is clear increase in total pressure over a large radial extent for rotor (ws) and rotor (wps) compared to rotor (wos). For $\phi=0.42$, as it is near stall flow coefficient, total pressure decreases for rotor (ws) and rotor (wps), rotor (wps) being worse. The effect of shrouding is felt over a large radial extent revealing the high three dimensionality of flows 


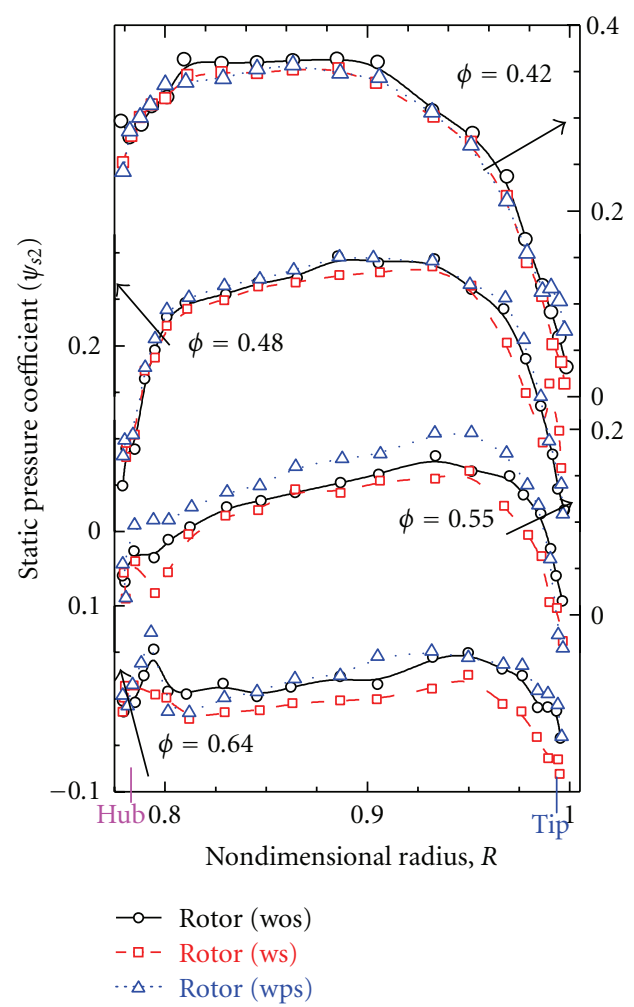

Figure 6: Radial distribution of static pressure coefficient at the rotor exit.

in the low-aspect-ratio compressors. The increase in total pressure is expected because the leakage jet located in the highly viscous region tends to diffuse rapidly. The partial shroud attached to the tip of the blade restricts the leakage flow from the pressure side to the suction side. Hence leakage flow interaction with the passage flow near the tip suction surface takes place further downstream where its harmful effects are reduced. But at $\phi=0.42$, the incoming flow near the tip is highly skewed. In case of unshrouded rotor, the leakage flow energises the boundary layer preventing it separating from the annulus wall. But in the shrouded cases, the leakage flow is being restricted resulting in separated casing wall boundary layer, producing higher losses instead of reducing them. The beneficial effects of partial shrouds can be probably retained by starting partial shrouds at about $20 \%$ chord from the leading edge, where the tip leakage flows originate.

Static Pressure Coefficient, $\psi_{s 2}$. The distribution of static pressure coefficients for the four flow coefficients and for the three configurations at the rotor exit is presented in Figure 6. Static pressure increased for $\phi=0.64,0.55$, and 0.48 for rotor (wps) compared to that of rotor (wos). Static pressure for rotor (ws) is reduced for $\phi=0.64$ and almost the same static pressure is observed for $\phi=0.55$ and 0.48 compared to that of rotor (wos). But for $\phi=0.42$, static pressure is reduced for both rotor (wps) and rotor (ws) compared to that of rotor (wos). This trend can be explained as follows: For all flow coefficients (except for $\phi=0.42$ ), total pressure

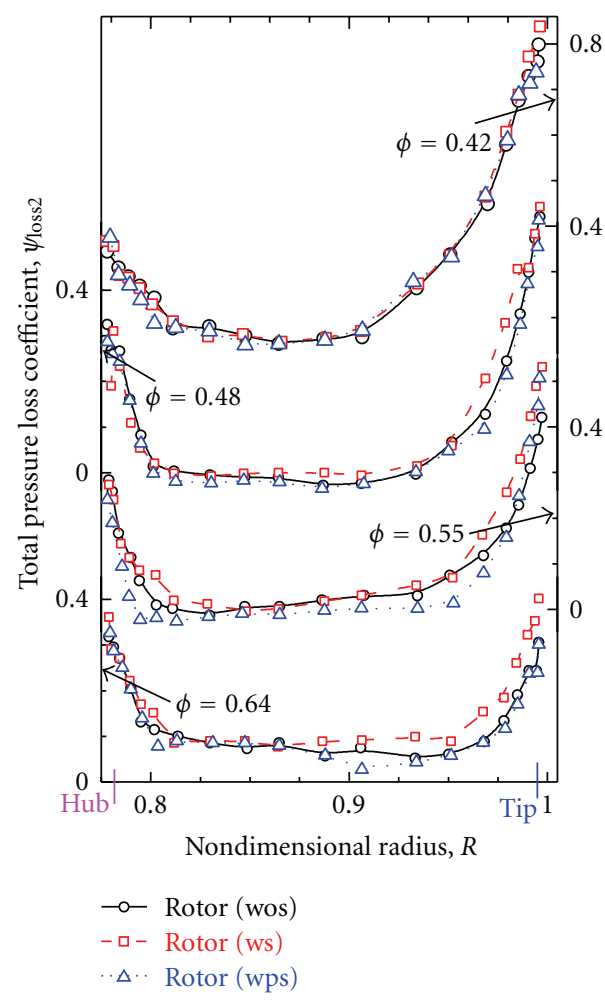

FIGURE 7: Radial distribution of total pressure loss coefficient at the rotor exit.

is higher for rotor (ws) than that of rotor (wos). Leakage flow acts in opposite direction of secondary flows. But partial shroud restricts leakage flow, reducing the scope of decreasing secondary flows and losses. Hence the static pressure is not in par with the total pressure rise. But in the case of rotor (wps), a small amount of leakage flow is released which has reduced the secondary flows and losses to a certain extent and increases static pressure rise. But for $\phi=0.42$, the total pressure itself is lower, increase in static pressure cannot be expected. For rotor (wps), though the total pressure is lower than that of rotor (wos), static pressure is same as that of rotor (wos).

Total Pressure Loss Coefficient, $\psi_{\text {loss } 2}$. The spanwise variation of total pressure loss coefficient for the four flow coefficients and for the three configurations at the rotor exit is shown in Figure 7. The total pressure loss coefficient is defined as follows:

$$
\psi_{\text {loss } 2}=\frac{2\left(\rho U_{2} C_{2 u}-P_{o 2}\right)}{\rho U_{c}^{2}},
$$

where $P_{\mathrm{o} 2}$ is total pressure at rotor exit, $\mathrm{Pa}, U_{2}$ is rotor speed, $\mathrm{m} / \mathrm{s}, U_{c}$ is rotor speed corresponding to the casing radius, $\mathrm{m} / \mathrm{s}$, and $C_{2 u}$ is tangential velocity at the rotor exit, $\mathrm{m} / \mathrm{s}$.

It can be seen that the losses are high in the annulus wall boundary layers and loss coefficient increases in the tip regions as the loading increases. The loss coefficient is minimum at the design flow coefficient. For flow coefficients, $0.64,0.55$, and 0.48 , the loss coefficient is lowest for rotor 


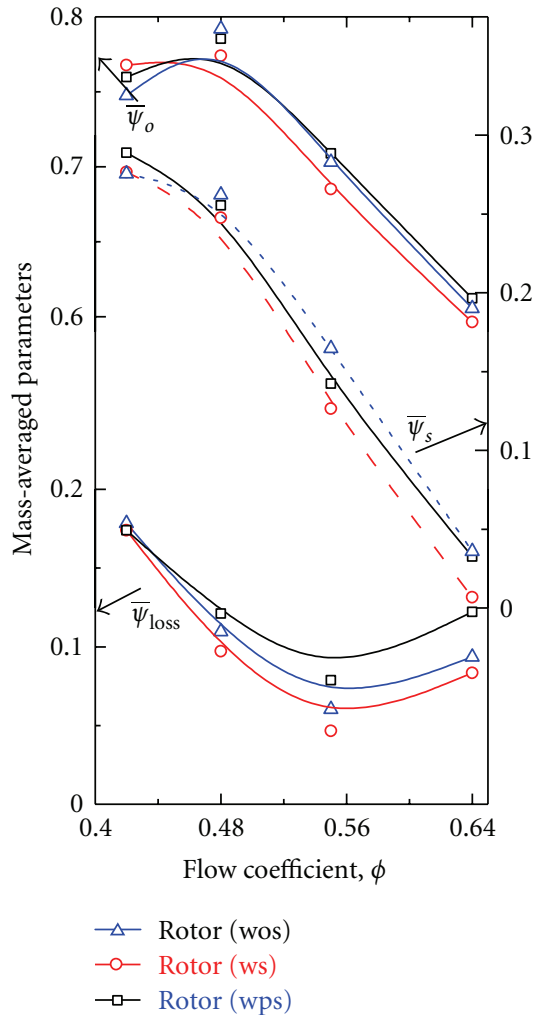

FIGURE 8: Mass-averaged performance of the Low-Aspect-Ratio Axial-Flow Fan Rotor.

(wps) configuration. At $\phi=0.42$, the loss coefficient is more or less the same for all the configurations.

4.1.2. Mass-Averaged Flow Parameters. The mass-averaged flow parameters with respect to the flow coefficients at the rotor exit are presented in Figure 8. The mass-averaged flow parameters are calculated as follows:

$$
\bar{q}=\frac{\int_{r_{h}}^{r_{c}} 2 \pi r q C_{x} d r}{\int_{r_{h}}^{r_{c}} 2 \pi r C_{x} d r},
$$

where $q$ represents any flow quantity and $\bar{q}$ is its massaveraged value across the annulus.

The total pressure is higher for rotor (ws) and rotor (wps) compared to that for rotor (wos) at $\phi=0.64$. For $\phi=0.55$ and 0.48 , the total pressure is highest for rotor (wps) followed by rotor (ws). Near the stall flow coefficient, that is, at $\phi=$ 0.42 , the total pressure is maximum for rotor (wos) and it is minimum for rotor (wps). It can be inferred that though the partial shrouds increase peak pressure rise, stall occurs at a higher flow coefficient, reducing the useful operating range.

From Figure 8, it can be seen that there is a definite increase in static pressure for rotor (wps) configuration for flow coefficients of $0.64,0.55$, and 0.48 at the rotor exit. In case of rotor (ws), though the total pressure is higher than that of rotor (wos), static pressure is less at the rotor exit. Near stalling flow coefficient, that is, at $\phi=0.42$, the static pressure is maximum for rotor (wos). The values of the mass

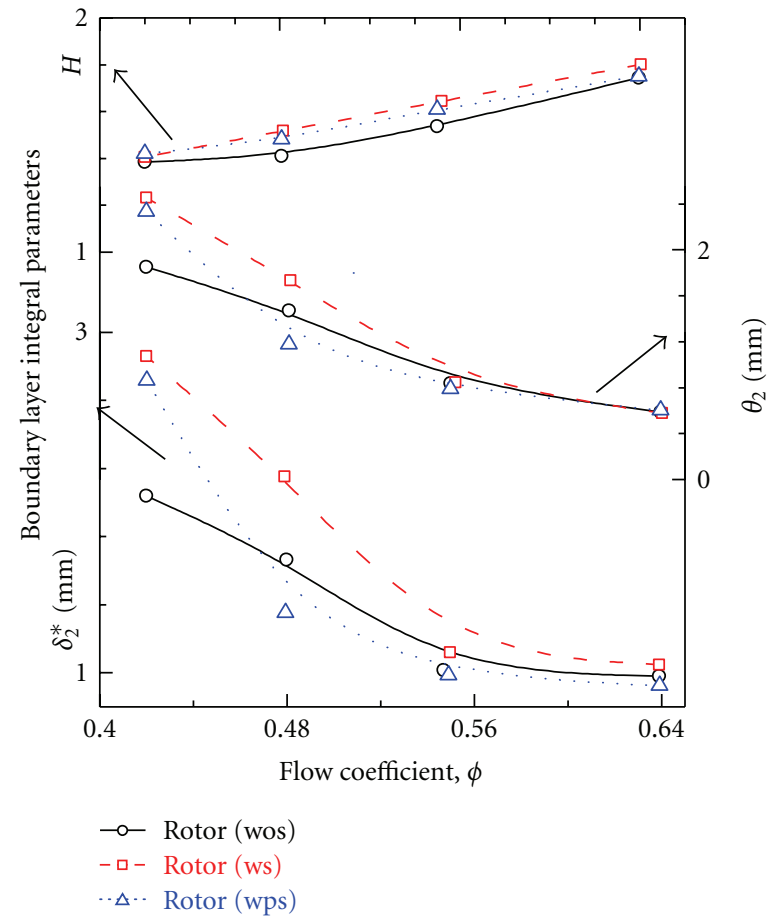

FIGURE 9: Variation of boundary layer integral parameters with flow coefficient at the rotor exit.

averaged parameters for the three configurations are tabulated in Table 5. Percentage increase in the mass averaged values is also given in Table 5 .

4.1.3. Boundary Layer Integral Parameters. The measured data from the pressure probe are used to determine the boundary layer integral parameters. The boundary layer integral parameters, namely, displacement thickness, $\delta^{*}$, momentum thickness, $\theta$, and shape factor, $H$, for the casing wall boundary layers are defined as follows:

$$
\begin{gathered}
\delta^{*}=\frac{\int_{\left(r_{c}-\delta\right)}^{r_{c}}\left(1-C_{x} / C_{x e}\right) r d r}{\int_{\left(r_{c}-\delta\right)}^{r_{c}} r d r}, \\
\theta=\frac{\int_{\left(r_{c}-\delta\right)}^{r_{c}}\left(1-C_{x} / C_{x e}\right)\left(C_{x} / C_{x e}\right) r d r}{\int_{\left(r_{c}-\delta\right)}^{r_{c}} r d r}, \\
H=\frac{\delta^{*}}{\theta} .
\end{gathered}
$$

Subscript $e$ refers to value at the edge of the boundary layer. The displacement and momentum thicknesses are based on the axial velocity rather than the absolute velocity.

The boundary layer integral parameters at the rotor exit are presented in Figure 9. The displacement thickness increases as the flow coefficient decreases, indicating thicker boundary layers. Its magnitude is more for rotor (ws) and is less for rotor (wps) compared to rotor (wos) for the three flow coefficients, $\phi=0.64,0.55$, and 0.48 . This may be due to the washing out of the boundary layer by the tip leakage vortex jet emerging from the suction surface in the case 
TABLE 5: Comparison of mass averaged values.

(a)

\begin{tabular}{lccccc}
\hline & Rotor (wos) & Rotor (ws) & Rotor (wps) & Rotor (ws) & \multicolumn{2}{c}{ Rotor (wps) } \\
$\phi$ & $\bar{\psi}_{o}$ & $\bar{\psi}_{o}$ & $\bar{\psi}_{o}$ & $\Delta \bar{\psi}_{o}(\%)$ & -1.0 \\
\hline 0.42 & 0.7677 & 0.7598 & 0.7475 & 1.4 & -2.6 \\
0.48 & 0.7741 & 0.7853 & 0.7921 & 3.4 & 2.3 \\
0.55 & 0.6852 & 0.7088 & 0.7031 & 2.6 & 2.6 \\
0.64 & 0.5965 & 0.6122 & 0.6055 & 1.5 \\
\hline
\end{tabular}

where $\Delta \bar{\psi}_{o}(\%)=\left(\bar{\psi}_{o}(\operatorname{rotor}(\right.$ ws $))-\bar{\psi}_{o}($ rotor $($ wos $\left.))\right) /\left(\bar{\psi}_{\mathrm{o}}(\right.$ rotor $($ wos $\left.))\right)$ for rotor (ws)

and $\Delta \bar{\psi}_{o}(\%)=\left(\bar{\psi}_{o}(\right.$ rotor $($ wps $))-\bar{\psi}_{o}($ rotor $($ wos $\left.))\right) /\left(\bar{\psi}_{\mathrm{o}}(\right.$ rotor $($ wos $\left.))\right)$ for rotor (wps).

(b) Similar definitions are used to calculate $\Delta \bar{\psi}_{S}$ and $\Delta \bar{\psi}_{\text {loss }}$.

\begin{tabular}{lccccc}
\hline & Rotor (wos) & Rotor (ws) & Rotor (wps) & Rotor (ws) & \multicolumn{2}{c}{ Rotor (wps) } \\
$\phi$ & $\bar{\psi}_{S}$ & $\bar{\psi}_{S}$ & $\bar{\psi}_{S}$ & $\Delta \bar{\psi}_{S}(\%)$ & -4.3 \\
\hline 0.42 & 0.2889 & 0.2765 & 0.2754 & -3.1 & -4.7 \\
0.48 & 0.2554 & 0.2476 & 0.2622 & -11.0 & 2.7 \\
0.55 & 0.1422 & 0.1265 & 0.1647 & -79.4 & 15.8 \\
0.64 & 0.0325 & 0.0067 & 0.0359 & & 10.4 \\
\hline
\end{tabular}

(c)

\begin{tabular}{|c|c|c|c|c|c|}
\hline & Rotor (wos) & Rotor (ws) & Rotor (wps) & Rotor (ws) & Rotor (wps) \\
\hline$\phi$ & $\bar{\psi}_{\text {loss }}$ & $\bar{\psi}_{\text {loss }}$ & $\bar{\psi}_{\text {loss }}$ & $\Delta \bar{\psi}_{\text {loss }}(\%)$ & $\Delta \bar{\psi}_{\text {loss }}(\%)$ \\
\hline 0.42 & 0.03112 & 0.03684 & 0.02541 & 18.4 & -18.3 \\
\hline 0.48 & 0.05139 & 0.05365 & 0.04565 & 4.4 & -11.2 \\
\hline 0.55 & 0.06262 & 0.06492 & 0.05577 & 3.7 & -10.9 \\
\hline 0.64 & 0.05473 & 0.05359 & 0.05015 & -2.1 & -8.4 \\
\hline
\end{tabular}

of rotor (wos). In case of rotor (ws), the partial shroud obstructing the leakage jet to wash away the boundary layer. In case of rotor (wps), the perforations in the shroud allow some amount of leakage flow. For $\phi=0.42$, the magnitudes of displacement thickness for the rotors with the partial shroud are higher compared to those for rotor (wos). At this flow coefficient, which is near stalling flow coefficient, the leakage flow allowed through the perforations may not be enough to reduce the boundary layer growth. The early stalling of the rotors with partial shrouds described earlier can be attributed to this boundary layer growth. Similar trends are observed for momentum thickness also. The magnitude of shape factor is less than 2, indicating that the boundary layers are turbulent, not separating.

4.2. Periodic Flow Measurements. From the steady state measurements, it is evident that rotor (wps) has improved performance compared to the other two configurations. Hence further experimental investigations are undertaken to measure the exit flow of rotor (wos) and rotor (wps) using a single slanted sensor hotwire probe in multiple positions. The measurements are carried out at three flow coefficients, namely, $\phi=0.48,0.55$, and 0.64 . For the sake of brevity, only results at the design flow coefficient, namely, $\phi=0.55$, are presented below.

4.2.1. Axial Velocity, $C_{2 x}$. Contours of the nondimensional axial velocity at $\phi=0.55$ (design flow coefficient) for rotor

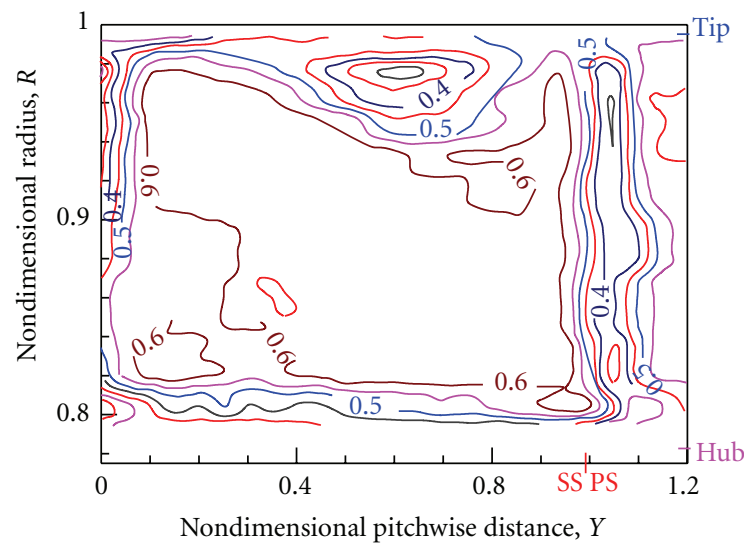

FIGURE 10: Axial velocity contours at the exit of rotor (wos) for $\phi=$ 0.55 .

(wos) and rotor (wps) are shown in Figures 10 and 11, respectively. There is a low velocity region near the tip forming a vortex in the passage for rotor (wos). The blade tip is immersed within the casing wall boundary layer hence, the tip leakage jet moves farther towards the pressure surface due to the blade motion. This leakage jet interacts with the main flow and mixing region has very low velocities and behaves like a separated zone. From the figure it can be seen that this region has occupied up to $R=0.94$. There is no such region in case of rotor (wps) configuration. Though the casing wall boundary layer is circumferentially nonuniform, the core 


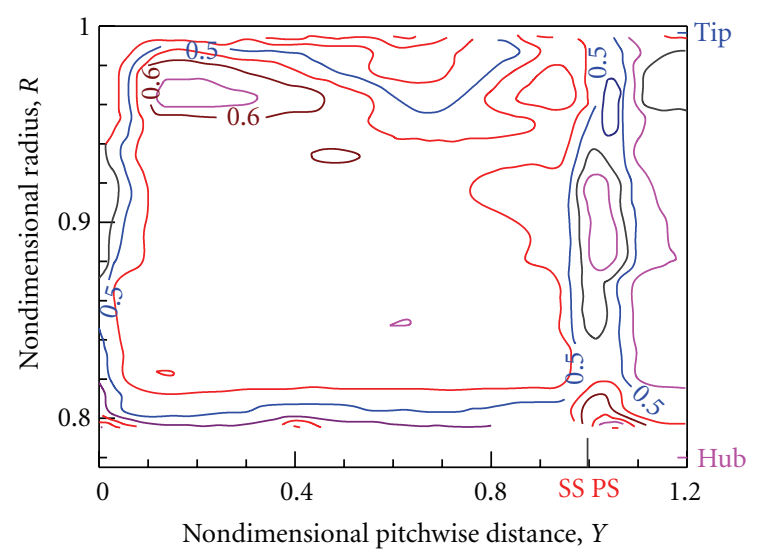

FIGURE 11: Axial velocity contours at the exit of rotor (wps) for $\phi=$ 0.55 .

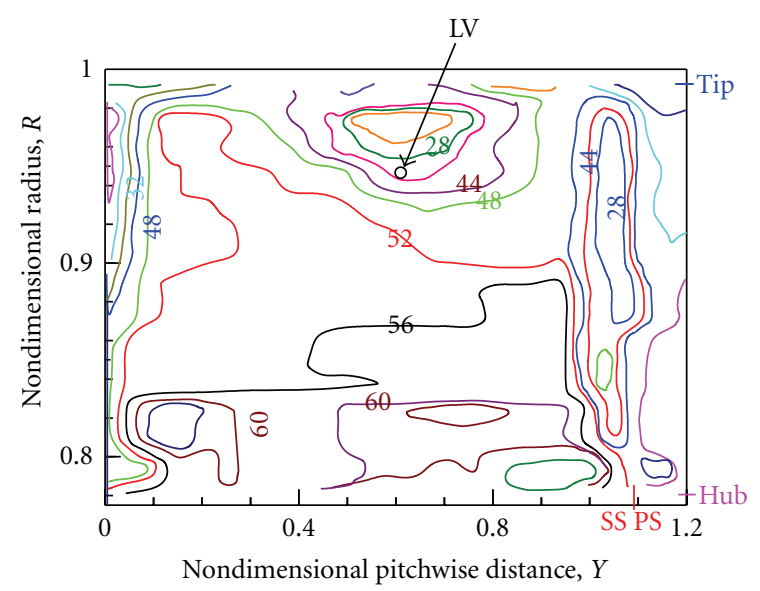

FIGURE 12: Relative flow angle contours at the exit of rotor (wos) for $\phi=0.55$.

of low velocity region is absent in case of rotor (wps) configuration. This is because the partial shroud attached to the tip of the blade restricts the fluid leakage through the clearance from the pressure side to the suction side.

The remaining portion of the blade passage has almost uniform flow except in the blade wake region and in the hub boundary layer. The casing wall boundary layer is nonuniform across the blade pitch. There is no evidence of thickening of the blade wake in the tip region.

4.2.2. Relative Flow Angle, $\beta_{2}$. Contours of the relative flow angle at $\phi=0.55$ (design flow coefficient) for rotor (wos) and rotor (wps) are shown in Figures 12 and 13, respectively. The relative flow angles are measured with respect to the tangential direction. In the tip leakage flow region, the relative flow angles decrease as the axial velocity decreases faster than the tangential velocity. The location and extent of this low relative flow angle is same as that of the low axial velocity. Binder and Romey [23] devised a method to locate the vortex core from the contours of absolute flow angle at the exit of a turbine nozzle. Since then many investigators have been

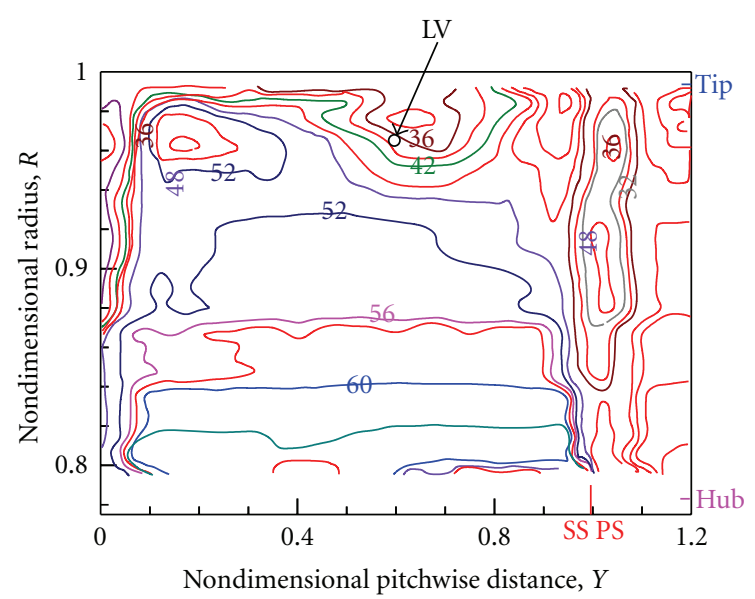

Figure 13: Relative flow angle contours at the exit of rotor (wps) for $\phi=0.55$.

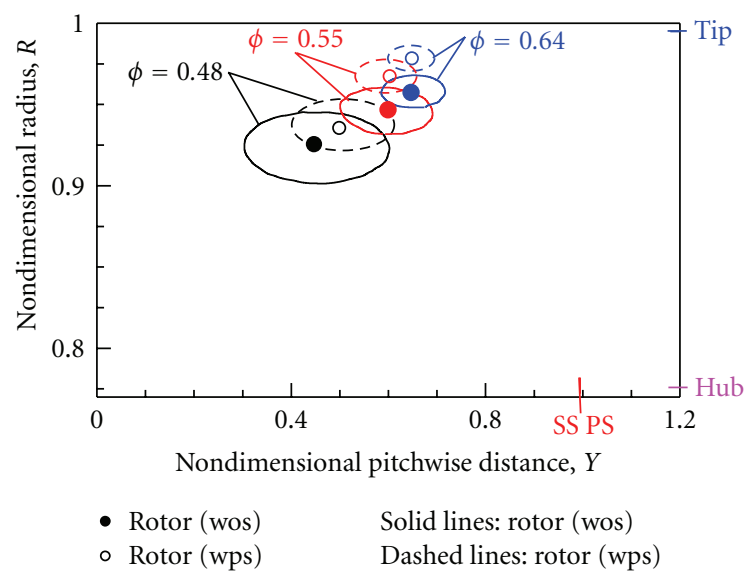

FIGURE 14: Location and extent of leakage vortex at different flow coefficients.

using this method to detect vortices at the exit of both stationary and rotating blade rows of various turbomachinery. This method is used here to detect leakage vortex in the present experiments. The centre of the vortex core is shown in Figures 11 and 12. Near the vortex centre, the lines are nearly straight, indicating a solid body type of vortex. Away from the vortex centre, the contours are oval indicating free vortex motion. The vortex core is also characterized by low axial velocities, as seen from the earlier figures.

But in case of rotor (wps), though the relative flow angle varies significantly in the tip region, the contours are not closely packed or straight. Hence it can be concluded that there is no solid body type of vortex. There is only a free vortex motion which in turn mixes out with the primary flow with minimum losses, giving higher static pressure at the stator exit. This can be attributed to the longer path which the leakage flow would have to travel over the width of the partial shroud during which it would be forced further downstream. Hence the interaction of the leakage flow with the passage flow near the tip region takes place further downstream where its harmful effects are reduced. 
4.2.3. Location and Extent of Tip Leakage Vortex. The location and extent of the tip leakage vortex at the rotor exit for the three flow coefficients and the two rotor configurations are shown in Figure 14. The leakage vortex is moving towards the pressure side and penetrating into the annulus as the flow coefficient is reduced. The spanwise and pitchwise extents of the tip leakage vortex also increase as the flow coefficient is reduced. This can be attributed to increase in the stage loading and hence stronger tip leakage vortex. But for rotor (wps) the extent is less than that of rotor (wos) for all the flow coefficients. The tip leakage vortex has to travel for a longer flow path over the partial shrouds provided on the tip of the rotor blades, thus weakening the tip leakage vortex.

\section{Conclusions}

The following major conclusions are drawn from the present experimental investigations.

5.1. From Pressure Probe Measurements. Of the three configurations studied, there is a definite improvement in total pressure obtained for rotor with partial shrouds. But only the rotor with partial shroud with perforations in the shape of discrete circular holes gives higher static pressure than that of the other two configurations. As there is no accumulation of leakage flow near the casing wall for rotors with partial shrouds, the boundary layer growth is reduced on the casing wall. Partial shrouds caused early stalling of the rotor. This is due to the separation of boundary layer on the casing wall for the rotors with shrouds at $\phi=0.42$, which otherwise would have been energized by the tip leakage flow.

5.2. From Periodic Flow Measurements. The leakage jet emerging from the tip clearance will not accumulate near the suction surface, and it travels towards the pressure surface. The mixing of the tip leakage jet with the main flow results in a region of low velocities which appears like a separated zone. This region of low velocities moved inside the annulus as the stage loading of the rotor is increased at lower flow coefficients. The region of low velocities is decreased considerably for rotor (wps).

The present experimental investigation is an initial attempt to improve performance of a low-aspect-ratio axialflow fan rotor using a fairly rough tip modification. Although performance of the rotor is improved at and above design conditions by means of the perforated partial shroud, stall margin is reduced. The most probable reason is the use of partial shroud of large width. The maximum width of the partial shroud is $11 \%$ of chord, while the maximum thickness of the blade at the tip is $5.5 \%$. It can be anticipated that performance and stall margin of the rotor may be improved by using improved partial shrouds, such as partial shrouds on pressure side only, partial shrouds having shorter chord than the blade at the tip, profiled partial shrouds, perforated partial shrouds, straight and inclined squealer tips, and so forth. Such successful attempts are reported in [14-17].

\section{Nomenclature}

$C_{x}$ : Axial velocity, $\mathrm{m} / \mathrm{s}$

$C_{u}$ : Tangential velocity, $\mathrm{m} / \mathrm{s}$

Ch: Blade chord, mm

$\mathrm{Ch}_{x}$ : Blade axial chord, $\mathrm{mm}$

$h$ : Blade height, $\mathrm{mm}$

$P$ : Pressure, $\mathrm{Pa}$

PS: Pressure surface

$R:$ Nondimensional radius, $r / r_{c}$

$r$ : Radius, mm

S: $\quad$ Blade spacing, $\mathrm{mm}$

SS: Suction surface

$t$ : Tip clearance, $\mathrm{mm}$

$U: \quad$ Blade peripheral speed, $\mathrm{m} / \mathrm{s}$

$V: \quad$ Volume flow rate, $\mathrm{m}^{3} / \mathrm{s}$

$X: \quad$ Nondimensional axial distance, $x / \mathrm{Ch}_{x}$

$x$ : Axial distance from rotor blade leading edge, $\mathrm{mm}$

$Y: \quad$ Nondimensional pitchwise distance, $y / S$

$y: \quad$ Pitchwise distance, $\mathrm{mm}$

$\beta$ : $\quad$ Relative flow angle, Deg

$\delta$ : $\quad$ Boundary layer thickness, $\mathrm{mm}$

$\delta^{*}:$ Displacement thickness, $\mathrm{mm}$

$\phi: \quad$ Flow coefficient $=V /\left(\pi r_{c}^{2} U_{c}\right)$

$\gamma: \quad$ Stagger angle, Deg

$\theta: \quad$ Momentum thickness, $\mathrm{mm}$

$\rho: \quad$ Fluid density, $\mathrm{kg} / \mathrm{m}^{3}$

$\psi: \quad$ Pressure coefficient $=2 P /\left(\rho U_{c}^{2}\right)$

$\tau: \quad$ Tip clearance as a percentage of chord $=\mathrm{t} / \mathrm{Ch} 100$.

\section{Subscripts}

1: Rotor inlet

2: $\quad$ Rotor exit

3: Stator exit

b: Blade

c: Casing

e: Boundary layer edge

$h$ : Hub

loss: Loss

o: Total

R: $\quad$ Rotor

S: $\quad$ Stator

$s: \quad$ Static

$t$ : Tip.

\section{Superscript}

-: Mass-averaged value.

\section{Acknowledgments}

The authors gratefully acknowledge the financial support by the Aeronautics Research and Development Board (AR \& DB), DRDO, Ministry of Defence, Government of India under the project, "Tip Clearance Flows in Compressors" (Grant no. Aero/RD/134/100/10/91-92/701). Late Professor $\mathrm{N}$. Venkatrayulu initiated this project. This paper is dedicated to his memory. 


\section{References}

[1] I. H. Hunter and N. A. Cumpsty, "Casing wall boundary layer development through an isolated compressor rotor," ASME Journal of Engineering for Power, vol. 104, no. 4, pp. 805-817, 1982.

[2] B. Lakshminarayana, M. Pouagare, and R. Davino, "Threedimensional flow field in the tip region of a compressor rotor passage - part I: mean velocity profiles and annulus wall boundary layer," ASME Journal of Engineering for Power, vol. 104, no. 3, pp. 760-771, 1982.

[3] M. Inoue and M. Kuroumaru, "Structure of tip clearance flow in an isolated axial compressor rotor," ASME paper number 88-GT-251, 1988.

[4] B. Lakshminarayana, M. Zaccaria, and B. Marathe, "The structure of tip clearance flow in axial flow compressors," ASME Journal of Turbomachinery, vol. 117, no. 3, pp. 336-347, 1995.

[5] R. E. Peacock, "Turbomachinery tip gap aerodynamics," in Proceedings of the 9th International Symposium on Air Breathing Engines, pp. 549-559, Athens, Greece, September 1989.

[6] H. Takata and Y. Tsukuda, "Stall margin improvement by casing treatment- its mechanism and effectiveness," ASME Journal of Engineering for Power, vol. 99, no. 1, pp. 121-133, 1977.

[7] C. H. Law, A. J. Wennerstrom, and W. A. Buzzell, "The use of vortex generators as inexpensive compressor casing treatment," SAE Paper, no. 760925, 1976.

[8] D. C. Wisler and B. F. Beacher, "Improved compressor performance using recessed clearance (trenches)," AIAA Journal of Propulsion and Power, vol. 5, no. 4, pp. 469-475, 1989.

[9] S. D. Hill, R. L. Elder, and A. B. McKenzie, "Application of casing treatment to an industrial axial-flow fan," Proceedings of the Institution of Mechanical Engineers, Part A, vol. 212, no. 4, pp. 225-233, 1998.

[10] H. Khaleghi and J. A. Teixeira, "Numerical study of discrete tip injection in a transonic axial compressor," in Proceedings of the ASME Turbo Expo Turbine Technical Conference, no. GT201023608, pp. 525-535, Glasgow, UK, June 2010.

[11] J. Giridhar, R. C. Murray, K. Essenhigh et al., "Control of tipclearance flow in a low speed axial compressor rotor with plasma actuation," in Proceedings of the ASME Turbo Expo Turbine Technical Conference, no. GT2010-22345, pp. 161-172, Glasgow, UK, June 2010.

[12] K. V. Patel, "Research on a high work axial gas generator turbine," SAE Paper, no. 800618, 1980.

[13] M. Ishida, H. Ueki, and Y. Senoo, "Effect of blade tip configuration on tip clearance loss of a centrifugal impeller," ASME Journal of Turbomachinery, vol. 112, no. 1, pp. 14-18, 1990.

[14] A. Corsini, F. Rispoli, and A. G. Sheard, "Shaping of tip endplate to control leakage vortex swirl in axial flow fans," ASME Journal of Turbomachinery, vol. 132, no. 3, pp. 1-9, 2010.

[15] A. Akturk and C. Camci, "Axial flow fan tip leakage flow control using tip platform extensions," ASME Journal of Fluids Engineering, vol. 132, no. 5, pp. 0511091-05110910, 2010.

[16] A. Akturk and C. Camci, "Tip clearance investigation of a ducted fan used in VTOL UAVs, part 1: baseline experiments and computational validation," in Proceedings of the ASME Turbo Expo Turbine Technical Conference, no. GT2011-46356, Vancouver, Canada, June 2011.

[17] A. Akturk and C. Camci, "Tip clearance investigation of a ducted fan used in VTOL UAVs, part 2: novel treatments via computational design and their experimental validation," in Proceedings of the ASME Turbo Expo Turbine Technical Conference, no. GT2011-46359, Vancouver, Canada, June 2011.
[18] A. J. Wennerstrom, "Low aspect ratio axial flow compressors: why and what it means," ASME Journal of Turbomachinery, vol. 111, no. 4, pp. 357-365, 1989.

[19] H. H. Brunn, A. Fitouri, and M. K. Khan, "The use of multiposition single yawed hot wire probe for measurements in swirling flow," ASME FED 167, Thermal Anemometry, pp 5765, 1993.

[20] B. Lakshminarayana, "Techniques for aerodynamics and turbulence measurements in turbomachinery rotors," ASME Journal of Engineering for Power, vol. 103, no. 2, pp. 374-392, 1981.

[21] R. J. Moffat, "Describing the uncertainties in experimental results," Experimental Thermal and Fluid Science, vol. 1, no. 1, pp. 3-17, 1988.

[22] G. Ch. V. Siva Kumar, Effect of partial shrouds on the performance and flow field of a low aspect ratio axial flow fan rotor, M.S. thesis, IIT Madras, 1987.

[23] A. Binder and R. Romey, "Secondary flow effects and mixing of the wake behind a turbine stator," ASME Journal of Engineering for Power, vol. 105, no. 1, pp. 40-46, 1983. 

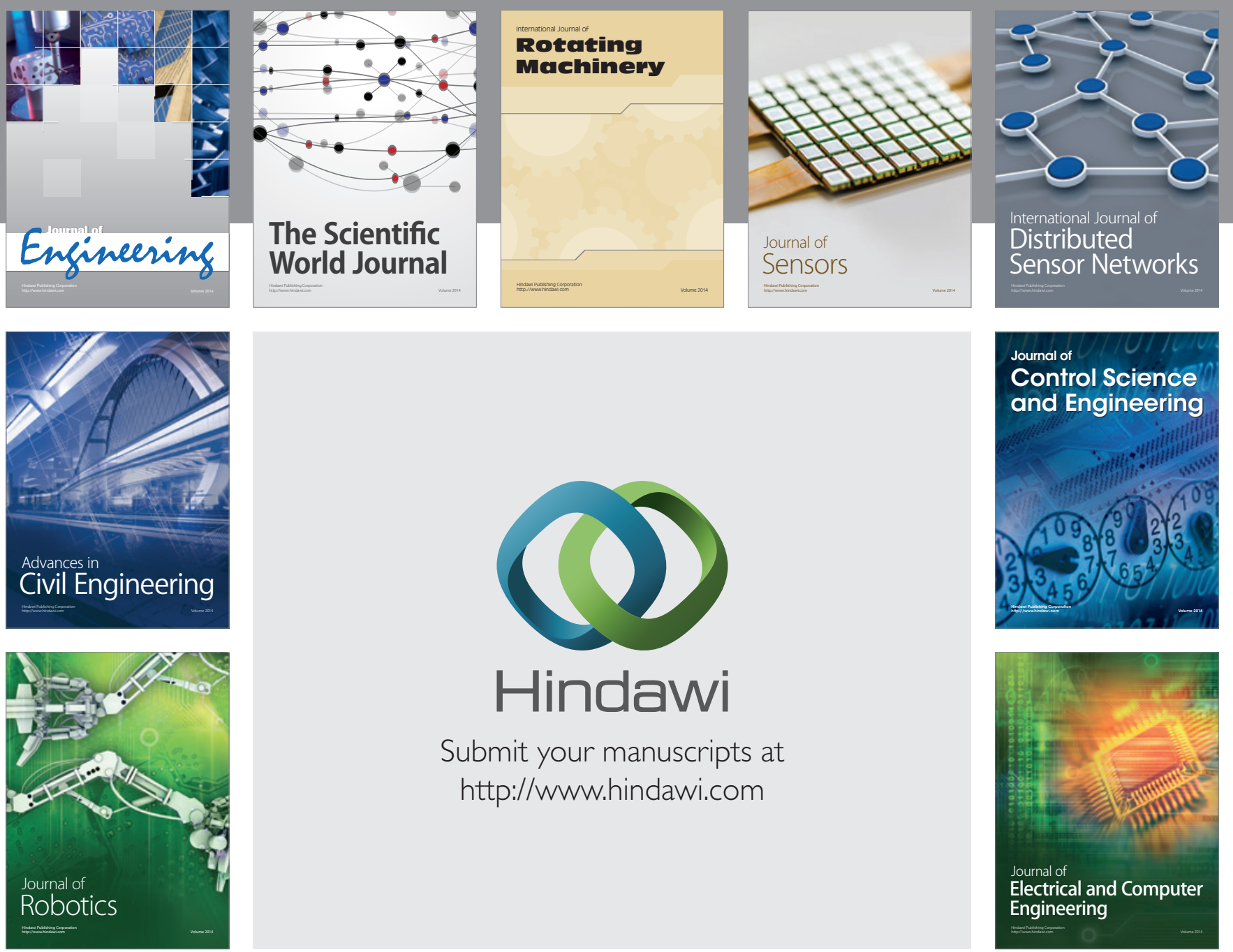

Submit your manuscripts at

http://www.hindawi.com
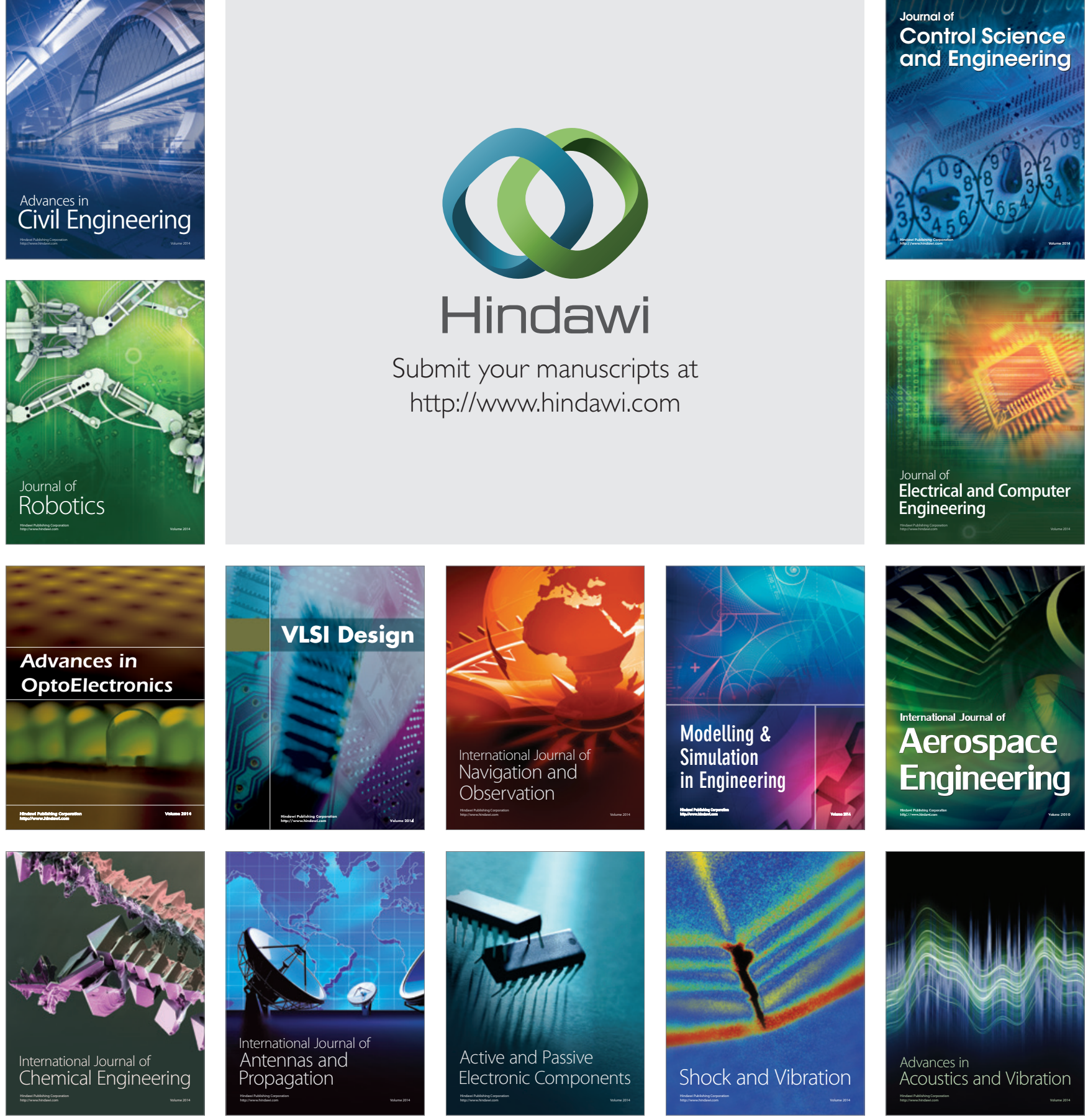